\title{
Phase separation of a multiple occupancy lattice gas
}

\author{
Reimar Finken, Jean-Pierre Hansen and Ard A Louis \\ Department of Chemistry, University of Cambridge, Cambridge, CB2 1EW, UK \\ E-mail: rf227@cam.ac.uk
}

Received 10 October 2003

Published 7 January 2004

Online at stacks.iop.org/JPhysA/37/577 (DOI: 10.1088/0305-4470/37/3/003)

\begin{abstract}
A binary lattice gas model that allows for multiple occupancy of lattice sites, inspired by recent coarse-grained descriptions of solutions of interacting polymers, is investigated by combining the steepest descent approximation with an exploration of the multidimensional energy landscape and by Gibbs ensemble Monte Carlo simulations. The one-component version of the model, involving on site and nearest-neighbour interactions, is shown to exhibit microphase separation into two sub-lattices with different mean occupation numbers. The symmetric two-component version of the multiple occupancy lattice gas is shown to exhibit a demixing transition into two phases above a critical mean occupation number.
\end{abstract}

PACS number: 61.20.Ja

\section{Introduction}

Simple fluids are dominated by excluded volume effects, which, within lattice gas models, are accounted for by the single occupancy constraint, whereby each site on a lattice can be occupied by at most one molecule. Effective interactions between macromolecules or self-assembled aggregates in complex fluids, on the other hand, can be 'soft', i.e. lack an impenetrable core. A good example is the effective pair potential between the centres of mass $(\mathrm{CM})$ of interacting polymer coils, obtained by taking statistical averages over monomer conformations for fixed distances $r$ between the CMs [1,2]. Recent extensive simulations of self-avoiding walk polymers carried out over a wide range of concentrations show that the repulsive state-dependent effective $\mathrm{CM}$ pair potential is of roughly Gaussian shape, of width governed by the polymer radius of gyration, and of maximum amplitude $v(r=0) \approx k_{B} T$ [3]; this behaviour reflects the fractal nature of polymers in good solvent, leading to a low entropic cost at full overlap. Several other complex fluids have been shown to exhibit ultrasoft coarsegrained interactions, e.g. star polymers [4] or effective particles considered within dissipative particle dynamics [5]. 
The penetrability of the corresponding 'Gaussian core' (GC) model, $v(r)=\epsilon \exp \left(-r^{2}\right)$, leads to interesting phase behaviour at low temperatures $\left(T^{*}=k_{B} T / \epsilon \ll 1\right)$, [6,7], but under conditions relevant for polymer solutions $\left(T^{*} \approx 1\right)$, the model behaves like a 'mean field' fluid [8]. However, binary Gaussian core mixtures, characterized by different energy scales $\epsilon_{\alpha \beta}$ for the three types of pairs, lead to phase separation for moderate couplings [8-10]. This demixing, which occurs for purely repulsive, penetrable interactions, is of a very different nature than the usual phase separation of incompatible fluids, which is generally driven by the long-range attractive intermolecular forces.

In this paper we examine the simplest lattice gas representation of penetrable particles, in an effort to gain further insight into this novel class of phase transitions. The penetrable nature of the effective interaction is reflected by allowing multiple occupancy of each lattice site. The simplest version of the model involves only on-site interactions of particles of the two species, with different energy penalties for the different types of pairs. While a steepest descent estimate of the grand partition function predicts phase separation, it will be shown that a more accurate treatment reveals the spurious nature of this transition, as expected for an effectively zero-dimensional system. The addition of nearest-neighbour interactions leads to a genuine demixing transition, similar to that predicted for continuous versions of soft core mixtures examined earlier within the random phase approximation (RPA) [8-10]. We explore this behaviour by mean-field theories and Gibbs ensemble Monte Carlo simulations. We also study the topology of the energy landscape.

\section{The multi-occupancy model}

Consider a $d$-dimensional lattice of $L$ sites and coordination number $q$, which can accommodate particles of two species. The occupancy of each site is characterized by the two-component vector of integer occupation numbers $\mathbf{n}_{i}=\left(n_{1}^{(i)}, n_{2}^{(i)}\right), 1 \leqslant i \leqslant L$. Particles interact only when they are on the same site or on nearest-neighbour (n.n.) sites. The on-site and off-site couplings are characterized by $2 \times 2$ matrices of interaction energies $\epsilon_{\alpha \beta}$ and $\eta_{\alpha \beta}(1 \leqslant \alpha, \beta \leqslant 2)$. In terms of the occupation numbers, the energy of the system reads

$$
E\left(\left\{\mathbf{n}_{i}\right\}\right)=\frac{1}{2} \sum_{i} \mathbf{n}_{i}{ }^{\mathrm{t}} \cdot \boldsymbol{\epsilon} \cdot \mathbf{n}_{i}-\frac{1}{2} \sum_{i} \sum_{\alpha} \epsilon_{\alpha \alpha} n_{\alpha}^{(i)}+\sum_{\langle i j\rangle} \mathbf{n}_{i}{ }^{\mathrm{t}} \cdot \boldsymbol{\eta} \cdot \mathbf{n}_{j}
$$

where the last summation is over n.n. sites only. For given chemical potentials $\boldsymbol{\mu}=\left(\mu_{1}, \mu_{2}\right)$ of the two species, the grand partition function is

$$
\begin{aligned}
\Xi & =\sum_{\left\{\mathbf{n}_{i}\right\}} \frac{\exp \left\{\beta \sum_{i} \boldsymbol{\mu} \cdot \mathbf{n}_{i}\right\}}{\prod_{\alpha, i} n_{\alpha}^{(i)} !} \exp \left\{-\beta E\left(\left\{\mathbf{n}_{i}\right\}\right)\right\} \\
& =\sum_{\left\{\mathbf{n}_{i}\right\}} \prod_{i=1}^{L} \frac{e^{\beta \boldsymbol{\mu}^{*} \cdot \mathbf{n}_{i}}}{n_{1}^{(i)} ! n_{2}^{(i)} !} \exp \left\{-\beta\left[\frac{1}{2} \sum_{i} \mathbf{n}_{i}{ }^{\mathrm{t}} \cdot \boldsymbol{\epsilon} \cdot \mathbf{n}_{i}+\sum_{\langle i j\rangle} \mathbf{n}_{i}{ }^{\mathrm{t}} \cdot \boldsymbol{\eta} \cdot \mathbf{n}_{j}\right]\right\} .
\end{aligned}
$$

The first sum is over all possible distributions of occupation numbers of the $L$ sites and the effective chemical potentials $\mu_{\alpha}^{*}=\mu_{\alpha}-\epsilon_{\alpha \alpha} / 2, \mu^{*}=\left(\mu_{1}^{*}, \mu_{2}^{*}\right)$ have been introduced.

The main task of this paper is to approximately evaluate the grand partition function (2) as a function of the temperature $T\left(\beta=1 /\left(k_{B} T\right)\right)$ and of the chemical potentials $\mu_{1}^{*}$ and $\mu_{2}^{*}$ of the two species. Using the standard rules of statistical mechanics, this will lead directly to the pressure $P=k_{B} T \ln (\Xi) / V$ and to the mean occupation numbers $n_{1}^{*}$ and $n_{2}^{*}$, and hence to the composition of the mixture. Phase separation will be signalled by a discontinuous change of the mean occupation number for well-defined values of $P, \mu_{1}^{*}$ and $\mu_{2}^{*}$. We shall first consider 
the simplest version of the model which only involves on-site interactions (i.e. $\boldsymbol{\eta}=\mathbf{0}$ ), before examining the case including nearest-neighbour interactions.

\section{The case of on-site interactions only}

In the absence of couplings between particles on different sites, the grand partition function (2), with $\boldsymbol{\eta}=\mathbf{0}$, factorizes into $L$ identical single site functions $\Xi_{\text {site }}$ :

$$
\Xi=\left[\sum_{n_{1}, n_{2}=0}^{\infty} \frac{\exp \left(\beta \boldsymbol{\mu}^{*} \cdot \mathbf{n}\right)}{n_{1} ! n_{2} !} \exp \left(-\frac{1}{2} \mathbf{n}^{\mathrm{t}} \cdot \boldsymbol{\epsilon} \cdot \mathbf{n}\right)\right]^{L}=\Xi_{\text {site }}^{L}
$$

The single site partition sum is not tractable analytically. For sufficiently large chemical potentials, implying mean occupation numbers $n_{1}^{*}, n_{2}^{*} \gg 1$, the latter may be treated as continuous variables, and the sums in equation (3) replaced by integrals

$$
\Xi_{\text {site }} \approx \int_{n_{1}=0}^{\infty} \int_{n_{2}=0}^{\infty} \frac{\exp \left(\beta \boldsymbol{\mu}^{*} \cdot \mathbf{n}-\frac{1}{2} \mathbf{n}^{\mathrm{t}} \cdot \boldsymbol{\epsilon} \cdot \mathbf{n}\right)}{\Gamma\left(n_{1}+1\right) \Gamma\left(n_{2}+1\right)} \mathrm{d} n_{1} \mathrm{~d} n_{2} .
$$

The integrand is sharply peaked around the most probable occupation numbers $\mathbf{n}^{*}=\left(n_{1}^{*}, n_{2}^{*}\right)$. Adopting the standard steepest descent method, we expand the logarithm of the integrand around $\mathbf{n}^{*}$ to second-order in $\delta \mathbf{n}=\mathbf{n}-\mathbf{n}^{*}$, i.e.:

$$
\begin{gathered}
\beta \boldsymbol{\mu}^{*} \cdot \mathbf{n}-\frac{1}{2} \beta \mathbf{n}^{\mathrm{t}} \cdot \boldsymbol{\epsilon} \cdot \mathbf{n}-\sum_{\alpha=1,2} \ln \Gamma\left(n_{\alpha}+1\right) \approx \beta \boldsymbol{\mu}^{*} \cdot \mathbf{n}^{*}-\frac{1}{2} \beta \mathbf{n}^{* \mathrm{t}} \cdot \boldsymbol{\epsilon} \cdot \mathbf{n}^{*}-\sum_{\alpha} \ln \Gamma\left(n_{\alpha}^{*}+1\right) \\
+\left(\beta \boldsymbol{\mu}^{*}-\beta \mathbf{n}^{* \mathrm{t}} \cdot \boldsymbol{\epsilon}-\left(\begin{array}{l}
\psi\left(n_{1}^{*}+1\right) \\
\psi\left(n_{2}^{*}+1\right)
\end{array}\right)\right) \cdot \delta \mathbf{n}-\frac{1}{2} \delta \mathbf{n}^{\mathrm{t}} \cdot \boldsymbol{\sigma} \cdot \delta \mathbf{n}
\end{gathered}
$$

where $\psi(n)=\mathrm{d} \ln \Gamma(n) / \mathrm{d} n$ is the digamma function, and the covariance matrix $\sigma$ is given by

$$
\boldsymbol{\sigma}=\left(\begin{array}{cc}
\beta \epsilon_{11}+\psi^{(1)}\left(n_{1}^{*}+1\right) & \beta \epsilon_{12} \\
\beta \epsilon_{12} & \beta \epsilon_{22}+\psi^{(1)}\left(n_{2}^{*}+1\right)
\end{array}\right)
$$

with $\psi^{(1)}(n)=\mathrm{d} \psi(n) / \mathrm{d} n$ (trigamma function). The location of the maximum follows from the extremum condition

$$
\beta \boldsymbol{\mu}^{*}-\beta \mathbf{n}^{* \mathrm{t}} \cdot \boldsymbol{\epsilon}-\left(\begin{array}{l}
\psi\left(n_{1}^{*}+1\right) \\
\psi\left(n_{2}^{*}+1\right)
\end{array}\right)=\mathbf{0}
$$

which provides the relation between $\boldsymbol{\mu}^{*}$ and $\mathbf{n}^{*}$. Inserting the expansion (5) into the integrand in equation (4) and extending the integral to negative occupation numbers (which is again justified provided $n_{\alpha}^{*} \gg 1$ ), the resulting Gaussian integral is easily evaluated and leads directly to the following equation of state

$$
\begin{aligned}
\beta P v_{0}=n_{1}^{*} \psi( & \left.n_{1}^{*}+1\right)-\ln \Gamma\left(n_{1}^{*}+1\right)+n_{2}^{*} \psi\left(n_{2}^{*}+1\right) \\
& -\ln \Gamma\left(n_{2}^{*}+1\right)+\frac{1}{2} \mathbf{n}^{* \mathrm{t}} \cdot \boldsymbol{\epsilon} \cdot \mathbf{n}^{*}+\ln \left(\frac{2 \pi}{\sqrt{|\boldsymbol{\sigma}|}}\right)
\end{aligned}
$$

where $v_{0}=V / L$ is the volume per site.

In the special case $\boldsymbol{\epsilon}=\mathbf{0}$ the partition function (3) can be evaluated exactly to yield the ideal gas equation of state $\beta P v_{0}=\exp \left(\beta \mu_{1}\right)+\exp \left(\beta \mu_{2}\right)=\left\langle n_{1}\right\rangle+\left\langle n_{2}\right\rangle$. Using the Stirling approximation $\Gamma(1+n) \approx \sqrt{2 \pi n}\left(\frac{n}{e}\right)^{n}$, the asymptotic virial pressure given by the saddle point approximation (8) reduces to $\beta P v_{0}=n_{1}^{*}+n_{2}^{*}+1$ in the limit of large $\mathbf{n}$. The extra constant is in fact negligible in this limit and its origin can be traced to the approximation of the integrand by a Gaussian. If we take the Taylor expansion (5) to third-order (instead of 
second), then the cubic term in $\delta \mathbf{n}$ skews the Gaussian towards higher occupation numbers. The mean occupation numbers no longer coincide with the maximum position, but are slightly shifted to $\left\langle n_{i}\right\rangle=n_{i}^{*}+\frac{1}{2}$, while equation (8) remains unaffected. In this way the ideal gas equation of state $\beta P v_{0}=\left\langle n_{1}\right\rangle+\left\langle n_{2}\right\rangle$ is recovered. Since we are interested in the large $\langle n\rangle$ limit, we restrict ourselves from now on to the second-order expansion in equation (5).

The virial pressure in equation (8), derived on the basis of steepest descent, is thermodynamically inconsistent with the relation (7) between chemical potentials and mean occupation numbers (identical to their most probable values). If the latter is integrated, an expression for the pressure follows which coincides with equation (8), but without the logarithmic fluctuation term. The situation is reminiscent of the RPA treatment of continuous penetrable (e.g. Gaussian) core mixtures, where the virial and compressibility routes lead to equations of state similar in structure to equation (8) with (virial) and without (compressibility) the last term [7-10]. The two equations of state become identical in the high density limit pointing to the asymptotic 'mean-field fluid' nature of systems of penetrable particles.

In view of the above analogy, it is not surprising that the equation of state (8) leads to phase separation between sites with different mean occupation numbers when $\epsilon_{12}^{2}>\epsilon_{11} \epsilon_{22}$. This predicted phase separation is obviously spurious, because of the effectively zero-dimensional nature of the model when restriction is made to on-site couplings. The above steepest descent treatment has two obvious shortcomings. The first deficiency is the replacement of the discrete sums in equation (3) by an integral, as well as the extension of the lower bounds in the integral (4) to $-\infty$; these approximations are expected to break down for low mean occupation numbers $n_{\alpha}^{*}$, or equivalently for low chemical potentials $\mu_{\alpha}^{*}$. This deficiency can be easily removed by reverting from the integrals to discrete sums over an interval of values of $\mathbf{n}^{*}$ in the vicinity of the most probable values $\mathbf{n}^{*}$ and retaining the continuous integrals outside this interval. In the one-component case, the resulting single-site partition function reads

$$
\begin{aligned}
\Xi_{\text {site }} \approx \sum_{n=n_{\text {low }}}^{n_{\text {high }}-1} \frac{1}{n !} & \mathrm{e}^{\beta \mu^{*} n} \mathrm{e}^{-\frac{1}{2} \beta \epsilon n^{2}}+\frac{\exp \left\{n^{*} \psi\left(n^{*}+1\right)\right\}}{\Gamma\left(n^{*}+1\right)} \exp \left\{\frac{1}{2} \beta \epsilon n^{* 2}\right\} \sqrt{\frac{\pi}{2 \sigma}} \\
\times & {\left[\operatorname{erf}\left(\sqrt{\frac{\sigma}{2}}\left(n_{\text {low }}-n^{*}\right)\right)+\operatorname{erf}\left(\sqrt{\frac{\sigma}{2}} n^{*}\right)-\operatorname{erf}\left(\sqrt{\frac{\sigma}{2}}\left(n_{\text {high }}-n^{*}\right)\right)+1\right] . }
\end{aligned}
$$

where $n_{\text {low }}=\max \left(\left\lceil n^{*}\right\rceil-m, 0\right)$ and $n_{\text {high }}-1=\left\lceil n^{*}\right\rceil+m-1$, and the ceil function \lceil\rceil rounds to the nearest higher integer.

The steepest descent result (8) is recovered for $m=0$ and sufficiently high $n^{*}$, such that $\operatorname{erf}\left(n^{*} \sqrt{\sigma / 2}\right)=1$. Results for the equation of state are shown in figure 1. Equation (8) is seen to yield surprisingly accurate results, even at relatively low densities $\left(n^{*} \approx 1\right)$ and only breaks down in the $n^{*} \rightarrow 0$ limit.

A more fundamental shortcoming of the steepest descent method is that equation (7) in fact predicts two maxima $\mathbf{n}^{*}$, separated by a saddle point. The situation is pictured in figure 2 . The steepest descent method includes only the contribution of the highest (global) maximum of the integrand to the partition function (4). This approximation leads to a discontinuous jump of the composition vector $\mathbf{n}^{*}$ when the two maxima become equal and hence to a first-order phase transition. In reality, when the contributions of the two maxima of the configuration integral (4) are properly included, the mean occupation numbers $\left\langle n_{1}\right\rangle$ and $\left\langle n_{2}\right\rangle$ vary continuously with the chemical potentials of the two species, as illustrated in figure 3 , according to expectation. 


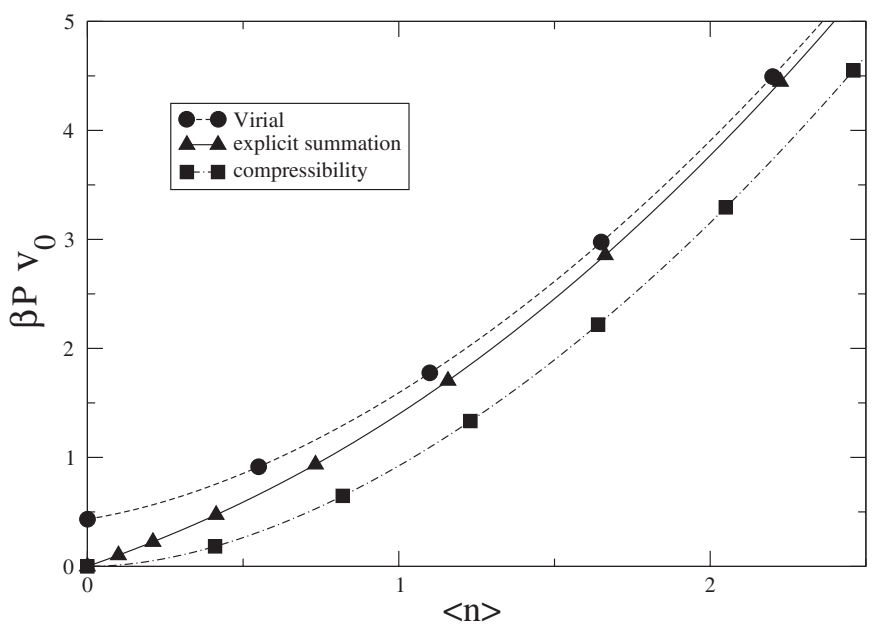

Figure 1. Equation of state of the one-component lattice gas with on site interaction energy $\epsilon=1 k_{B} T$. The normalized pressure $\beta P v_{0}$ is plotted as a function of the mean occupation number $\langle n\rangle$ of one cell. Shown are values obtained by using the steepest descent method (dashed curve, circles), the pressure consistent with the chemical potential (7) (dash-dotted line, squares) and the pressure obtained from the grand canonical partition sum by explicitly summing $2 m$ terms around the maximum of the Boltzmann factor as explained in the text with $m=100$ (continuous line, triangles). At this value the correction term in equation (9) is well below $10^{-5}$, so that the resulting pressure can be considered nearly exact. The steepest descent method is seen to be very accurate except, as expected, at low densities.

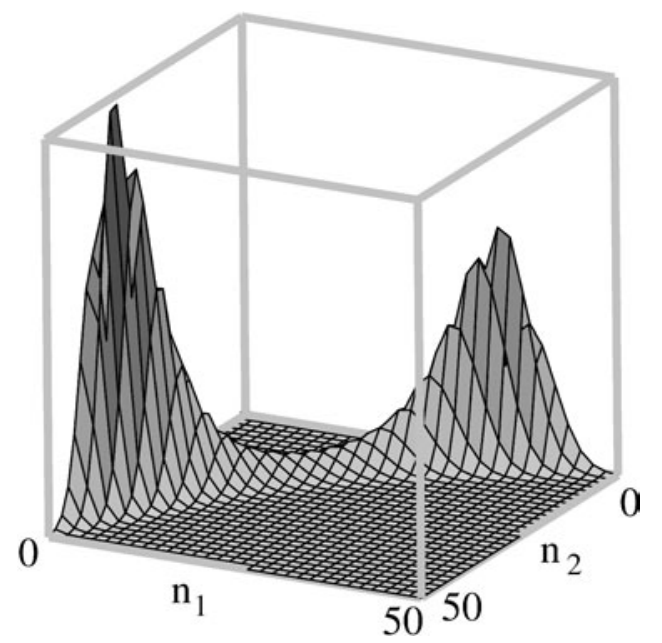

Figure 2. The Boltzmann factor $\exp (\beta \boldsymbol{\mu} \cdot \mathbf{n}-\beta E(\mathbf{n})) /\left(n_{1} ! n_{2} !\right)$ as a function of the mean occupation numbers $n_{1}, n_{2}$ for the lattice system with on site interaction $\epsilon_{11}=\epsilon_{22}=$ $0.1 k_{B} T, \epsilon_{12}=0.2 k_{B} T$ and no nearest-neighbour interaction, at the chemical potentials $\mu_{1}=$ $6.5 k_{B} T, \mu_{2}=6.0 k_{B} T$. The appearance of a secondary maximum is clearly seen.

\section{Including nearest-neighbour interactions}

We now consider the model defined by equations (1) and (2) and including n.n. interactions between particles of both species, i.e. $\boldsymbol{\eta} \neq \mathbf{0}$. The partition function (2) no longer factorizes 


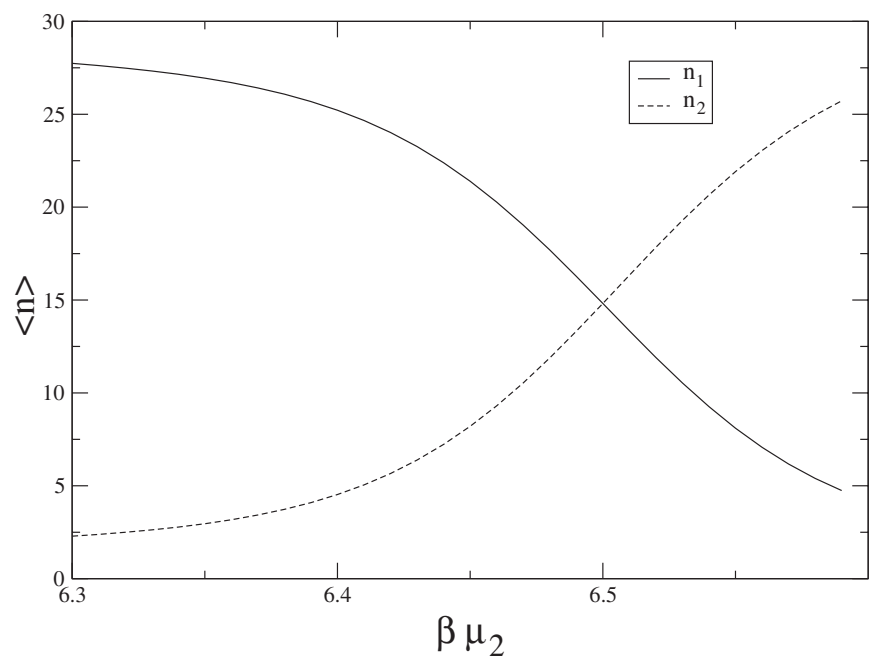

Figure 3. Mean occupation numbers $\left\langle n_{1}\right\rangle,\left\langle n_{2}\right\rangle$ in a symmetric binary lattice gas mixture for on-site interaction energies $\epsilon_{11}=\epsilon_{22}=0.1 k_{B} T \epsilon_{12}=0.2 k_{B} T$ and vanishing nearest neighbour interaction as a function of chemical potential $\mu_{2}$, keeping $\mu_{1}=6.5 k_{B} T$ fixed. The values were calculated using a simple generalization of equation (9). As expected, the occupation numbers vary continuously with chemical potentials in this effectively zero-dimensional system.

into single site sums. Proceeding as in section 3, we replace the discrete sums over occupation numbers by integrals and approximate the integrand by Gaussians centred on each of the local maxima. The latter are determined by a generalization of equation (7), namely

$$
\beta \boldsymbol{\mu}^{*}=\beta \mathbf{n}_{i}^{* \mathrm{t}} \cdot \boldsymbol{\epsilon}+\sum_{i \text { n.n.j }} \beta \mathbf{n}_{j}^{* \mathrm{t}} \cdot \boldsymbol{\eta}+\left(\begin{array}{l}
\psi\left(n_{1, i}^{*}+1\right) \\
\psi\left(n_{2, i}^{*}+1\right)
\end{array}\right) \quad 1 \leqslant i \leqslant L .
$$

In the limit $\boldsymbol{\eta} \rightarrow \mathbf{0}$, the $\mathbf{n}_{i}^{*}$ on different sites are independent and can each take two values, corresponding to the two maxima found in section 3 . When the n.n. interactions are switched on, the $\mathbf{n}_{i}^{*}$ are coupled so that a complex energy landscape emerges in $2 L$-dimensional occupation-number space, featuring a rapidly increasing number of maxima of the Boltzmann factor. By continuity one can still expect $2^{L}$ solutions to equations (10) in the most general case, at least for sufficiently weak coupling. The approximate grand partition function now reads

$$
\begin{aligned}
\Xi=\sum_{\left\{\mathbf{n}_{i}^{*}\right\}} \exp [ & \left.\frac{1}{2} \sum_{i} \mathbf{n}_{i}^{*} \cdot \boldsymbol{\epsilon} \cdot \mathbf{n}_{i}^{*}+\sum_{i, \alpha} n_{i, \alpha}^{*} \psi\left(n_{i, \alpha}^{*}+1\right)-\sum_{i, \alpha} \ln \Gamma\left(n_{i, \alpha}^{*}+1\right)+\sum_{\langle i j\rangle} \mathbf{n}_{i}^{*} \cdot \boldsymbol{\eta} \cdot \mathbf{n}_{j}^{*}\right] \\
& \times \int \mathrm{d} \delta \mathbf{n}_{1} \cdots \int \mathrm{d} \delta \mathbf{n}_{L} \exp \left(-\left[\frac{1}{2} \sum_{i} \delta \mathbf{n}_{i} \cdot \boldsymbol{\sigma}_{i} \cdot \delta \mathbf{n}_{i}+\sum_{\langle i j\rangle} \beta \delta \mathbf{n}_{i} \cdot \boldsymbol{\eta} \cdot \delta \mathbf{n}_{j}\right]\right)
\end{aligned}
$$

where the covariance matrices $\sigma_{i}$ are obvious generalizations of equation (6), with $n_{\alpha}^{*}$ replaced by $n_{\alpha, i}^{*}$.

The grand partition function is thus expressed as a sum over all 'configurations' $\left\{\mathbf{n}_{i}^{*}\right\}$ which maximize the integrand in the continuous representation generalizing equation (4), i.e. which satisfy the set of equations (10). The Gaussian fluctuation integrals in the second factor 
depend on the configuration $\left\{\mathbf{n}_{i}^{*}\right\}$ through the covariance matrices $\boldsymbol{\sigma}_{i}$. For given $\left\{\boldsymbol{\sigma}_{i}\right\}$ the coupled Gaussian integrals can be calculated explicitly by a standard normal mode analysis.

The locations of the local maxima $\mathbf{n}_{i}^{*}$ depend on the occupation numbers of the neighbouring cells $\mathbf{n}_{j}^{*}$ via (10). If this were not the case, then the partition function (11) would exactly factor into a product of a partition sum that is isomorphic to that of an Ising model (first factor in (11)) and the partition sum of a Gaussian model. The phase behaviour of a very similar system has been investigated in [11]. The model we examine can thus only be mapped onto an Ising (plus Gaussian) model in the limit of weak nearest-neighbour interactions $\boldsymbol{\eta}$.

In order to gain further insight, we consider the 'ground state', corresponding to the low temperature or strong coupling limit. In that limit all $\mathbf{n}_{i}^{*}$ corresponding to the lowest minimum on the total energy surface are expected to be equal $\left(\mathbf{n}_{i}^{*}=\mathbf{n}^{*}\right)$ and determined by a single extremum condition

$$
\beta \boldsymbol{\mu}^{*}=\beta \mathbf{n}^{* \mathrm{t}} \cdot(\boldsymbol{\epsilon}+q \boldsymbol{\eta})+\left(\begin{array}{l}
\psi\left(n_{1}^{*}+1\right) \\
\psi\left(n_{2}^{*}+1\right)
\end{array}\right)
$$

where $q$ is the coordination number of the lattice. In the low temperature limit only the contribution of this one configuration to the sum in equation (11) is taken into account. The second-factor in this equation then becomes invariant with respect to translations, so that the corresponding integral is best evaluated in Fourier space.

We therefore introduce the Fourier components of the density fluctuations

$$
\delta \tilde{\mathbf{n}}_{\mathbf{k}}=\frac{1}{L} \sum_{j} \delta \mathbf{n}_{j} \mathrm{e}^{\mathrm{ik} \cdot \mathbf{R}_{j}}
$$

where $\mathbf{r}_{j}$ denotes the position of the lattice site $j$ in units of the lattice constant. Assuming Born-von Karman boundary conditions the wave vector $\mathbf{k}$ can only assume discrete values. The total interaction energy can be written in Fourier space as

$$
\frac{1}{2} \sum_{i} \delta \mathbf{n}_{i} \cdot \boldsymbol{\sigma} \cdot \delta \mathbf{n}_{i}+\sum_{\langle i j\rangle} \beta \delta \mathbf{n}_{i} \cdot \boldsymbol{\eta} \cdot \delta \mathbf{n}_{j}=\frac{L}{2} \sum_{\mathbf{k}} \delta \tilde{\mathbf{n}}_{-\mathbf{k}} \cdot \mathbf{D}(\mathbf{k}) \cdot \delta \tilde{\mathbf{n}}_{\mathbf{k}}
$$

where the matrix $\mathbf{D}(\mathbf{k})$ is given by

$$
\mathbf{D}(\mathbf{k})=\boldsymbol{\sigma}+\beta \boldsymbol{\eta} \sum_{j \text { n.n. } 0} \mathrm{e}^{i \mathbf{k} \cdot \mathbf{R}_{j}}
$$

For a simple cubic lattice we obtain

$$
\mathbf{D}(\mathbf{k})=\sigma+2\left(\cos k_{x}+\cos k_{y}+\cos k_{z}\right) \beta \boldsymbol{\eta} .
$$

The Fourier modes of the density fluctuations thus decouple, so that the multiple integral in equation (11) can be evaluated in Fourier space as a product of Gaussian integrals

$$
\begin{aligned}
\int \mathrm{d} \delta \mathbf{n}_{1} \cdots & \int \mathrm{d} \delta \mathbf{n}_{L} \exp \left(-\left[\frac{1}{2} \sum_{i} \delta \mathbf{n}_{i} \cdot \boldsymbol{\sigma}_{i} \cdot \delta \mathbf{n}_{i}+\sum_{\langle i j\rangle} \beta \delta \mathbf{n}_{i} \cdot \boldsymbol{\eta} \cdot \delta \mathbf{n}_{j}\right]\right) \\
& =L^{L} \int \mathrm{d} \delta \tilde{\mathbf{n}}_{\mathbf{k}_{1}} \cdots \int \mathrm{d} \delta \tilde{\mathbf{n}}_{\mathbf{k}_{L}} \exp \left(-\frac{L}{2} \sum_{\mathbf{k}} \delta \tilde{\mathbf{n}}_{\mathrm{bf}-\mathrm{k}} \cdot \mathbf{D}(\mathbf{k}) \cdot \delta \tilde{\mathbf{n}}_{\mathbf{k}},\right) \\
& =(2 \pi)^{L} \prod_{\mathbf{k}} \frac{1}{\sqrt{|\mathbf{D}(\mathbf{k})|}} .
\end{aligned}
$$




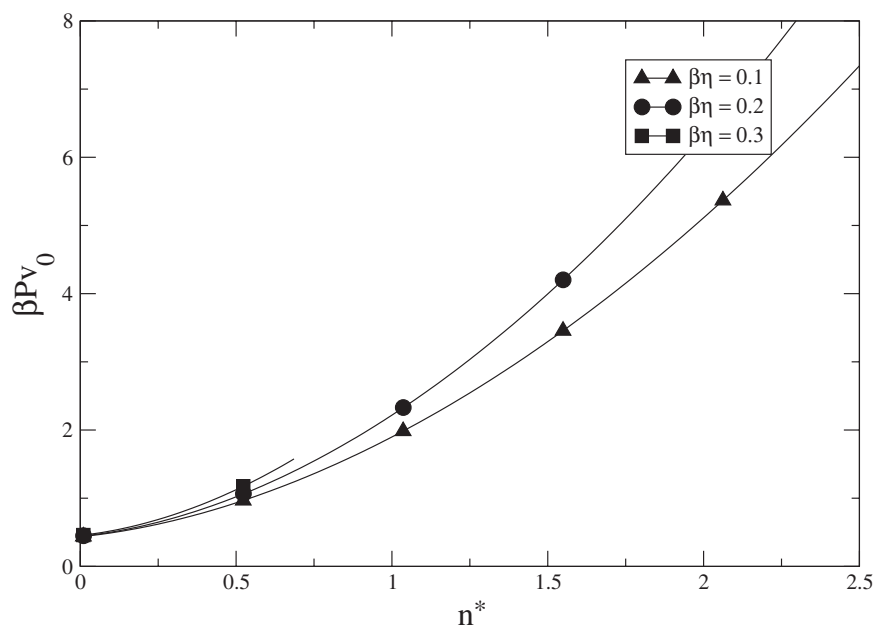

Figure 4. Virial equation of state of a one component lattice system with on site interaction $\epsilon=1 k_{B} T$ and nearest-neighbour interaction $\eta=0.1 k_{B} T$ (triangles), $\eta=0.2 k_{B} T$ (circles) and $\eta=0.3 k_{B} T$ (squares). For $\beta \eta>1 / 6$ our analysis predicts an instability of the homogeneous phase towards microphase separation beyond a certain density. For $\eta=0.1 k_{B} T$ this criterion is not obeyed and the fluid remains stable at all densities. For $\eta=0.2 k_{B} T$ the instability is at $n^{*}=4.48$ (outside the density range of this plot) and for $\eta=0.3$ it is at $n^{*}=0.69$.

The grand partition function then reduces to

$$
\begin{gathered}
\Xi=\left[\exp \left\{\frac{1}{2} \mathbf{n}^{*} \cdot(\boldsymbol{\epsilon}+q \boldsymbol{\eta}) \cdot \mathbf{n}^{*}+\sum_{\alpha} n_{\alpha}^{*} \psi\left(n_{i, \alpha}^{*}+1\right)-\ln \Gamma\left(n_{\alpha}^{*}+1\right)\right\}\right]^{L} \\
\times(2 \pi)^{L} \exp \left\{-\frac{L}{2} \int \frac{\mathrm{d} \mathbf{k}}{(2 \pi)^{3}} \ln (|\mathbf{D}(\mathbf{k})|)\right\}
\end{gathered}
$$

leading to the equation of state (e.o.s.)

$$
\begin{gathered}
\beta P v_{0}=\sum_{\alpha}\left[n_{\alpha}^{*} \psi\left(n_{\alpha}^{*}+1\right)-\ln \Gamma\left(n_{\alpha}^{*}+1\right)\right]+\frac{1}{2} \mathbf{n}^{*} \cdot(\epsilon+q \boldsymbol{\eta}) \cdot \mathbf{n}^{*}+\ln (2 \pi) \\
-\frac{1}{2} \int \frac{\mathrm{d} \mathbf{k}}{(2 \pi)^{3}} \ln (|\mathbf{D}(\mathbf{k})|) .
\end{gathered}
$$

In the limit of large occupation numbers $\left(n_{\alpha}^{*} \gg 1\right)$ and vanishing nearest-neighbour interaction $(\boldsymbol{\eta}=\boldsymbol{0})$ we recover equation (8). As in the case without nearest-neighbour interaction, there is a thermodynamic inconsistency between the e.o.s. (19) and the dependence of the chemical potentials on the occupation numbers (12). Integrating the latter would again lead to an e.o.s. similar in structure to equation (19), albeit without the final two logarithmic terms. The virial equation of state is shown in figure 4. For high nearest-neighbour interaction $(\epsilon-q \boldsymbol{\eta}$ no longer being positive definite) the fluctuation term diverges at $\mathbf{k}=(\pi, \pi, \pi)$. This instability indicates a transition of the ground state from the homogeneous phase to a microfluid phase where high and low occupation numbers alternate in a checkered fashion. To show that the microphase exists also for the one-component fluid (for $q \eta>\epsilon$ ) a canonical Monte Carlo simulation was run on a $10 \times 10 \times 10$ cubic lattice $(q=6)$ with the parameters $\epsilon=0.1 k_{B} T, \eta=0.033 k_{B} T$. Initially every lattice site was filled with 20 particles. After equilibration a histogram of the occupation numbers occurring over the next 1000000 steps 


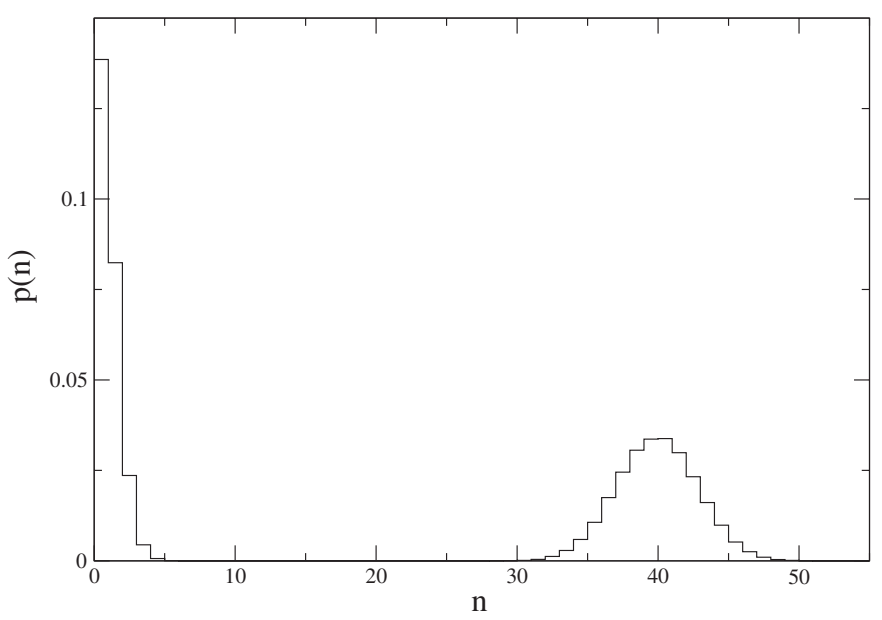

Figure 5. Histogram of the occupation numbers in a one component lattice system $(10 \times 10 \times 10)$ with $\epsilon=0.1 k_{B} T$ and $\eta=0.033 k_{B} T$. The probability $p(n)$ to find a lattice site with occupation number $n$ (averaged over 1000000 steps after equilibration) is shown. The canonical simulation was started with 20 particles on each lattice site. Because of the large nearest-neighbour interaction the initially homogeneous fluid develops into a microphase with a bimodal distribution of the occupation numbers.

was recorded. The result is shown in figure 5. It is clearly seen that the initial peak at $n=20$ has vanished and a bimodal distribiution around $n \approx 0$ and $n \approx 40$ has developed. The development of the microphase is easy to understand when we estimate and compare the energetic and entropic costs of the microphase with (mean) occupation numbers $n_{a}$ and $n_{b}$ with those of a homogeneous phase with average density $\bar{n}=\left(n_{a}+n_{b}\right) / 2$. The entropy of the microphase per site is $s^{\text {micro }}=k_{B}\left[n_{a} \ln n_{a}+n_{b} \ln n_{b}\right] / 2$, compared to that of the homogeneous phase $s^{\text {hom }}=k_{B} \bar{n} \ln \bar{n}$. The difference between the two is simply the mixing entropy of the ideal gas, thereby favouring the homogeneous density distribution. On the other hand the internal energy of the microphase per site is $u^{\text {micro }}=\epsilon n_{a}^{2} / 4+\epsilon n_{b}^{2} / 4+q \eta n_{a} n_{b} / 2$, compared to that of the homogeneous phase $u^{\text {hom }}=(\epsilon+q \eta) \bar{n}^{2} / 2$. The difference between the two internal energies is $u^{\text {micro }}-u^{\text {hom }}=(\epsilon-q \eta)\left(n_{b}-n_{a}\right)^{2} / 8$. For strong nearestneighbour interaction the microphase is therefore energetically favoured. The nature of the transition from the homogeneous state to the micro state will be investigated elsewhere. We note that a similar transition has been discussed by Likos et al for a continuous soft core system [12].

The explicit knowledge of the Fourier components of the density fluctuations allows us to evaluate the structure factor of the fluid. For $\mathbf{k} \neq \mathbf{0}$ we obtain

$$
\begin{aligned}
S_{\alpha \beta}(\mathbf{k})= & \frac{1}{n_{1}^{*}+n_{2}^{*}}\left\langle\tilde{\delta} n_{\alpha}(\mathbf{k}) \tilde{\delta n_{\beta}}(-\mathbf{k})\right\rangle \\
& =\frac{1}{n_{1}^{*}+n_{2}^{*}} \frac{\int \mathrm{d} \tilde{\delta}_{1}(\mathbf{k}) \int \mathrm{d} \tilde{\delta} n_{2}(\mathbf{k}) \tilde{\delta} n_{\alpha}(\mathbf{k}) \tilde{\delta n_{\beta}}(\mathbf{k}) \exp (-\tilde{\delta \mathbf{n}}(\mathbf{k}) \cdot \mathbf{D}(\mathbf{k}) \cdot \tilde{\delta \mathbf{n}}(\mathbf{k}))}{\int \mathrm{d} \tilde{\delta} n_{1}(\mathbf{k}) \int \mathrm{d} \tilde{\delta} n_{2}(\mathbf{k}) \exp (-\tilde{\delta \mathbf{n}}(\mathbf{k}) \cdot \mathbf{D}(\mathbf{k}) \cdot \tilde{\delta \mathbf{n}}(\mathbf{k}))} \\
& =\frac{1}{n_{1}^{*}+n_{2}^{*}}\left(\mathbf{D}^{-1}\right)_{\alpha \beta}(\mathbf{k}) .
\end{aligned}
$$

From the particle-particle structure factors $S_{\alpha \beta}$ the Bhatia-Thornton structure factors [14] can be extracted. These are compared with data from a canonical Monte Carlo simulation in 


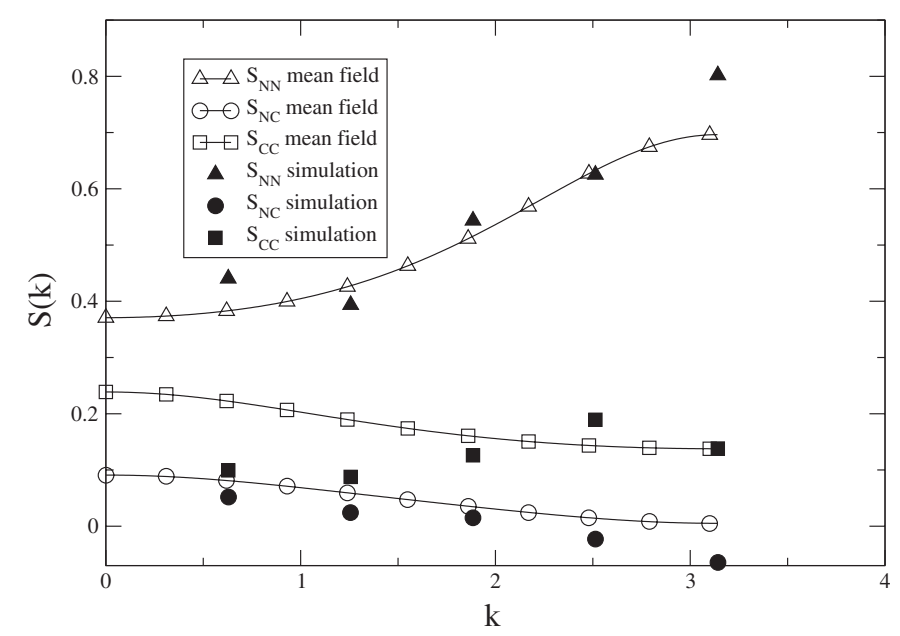

Figure 6. Bhatia-Thornton structure factors for the symmetric binary mixture with $\beta \epsilon_{11}=\beta \epsilon_{22}=$ $0.1, \beta \epsilon_{12}=0.2, \boldsymbol{\eta}=0.1 \epsilon$. The mean-field values (open symbols, lines) are compared to simulation data of a canonical Monte Carlo simulation (filled symbols) on a $10 \times 10 \times 10$ lattice. The densitydensity ( $S_{n n}$, triangles), density-concentration $\left(S_{\mathrm{nc}}\right.$, circles) and concentration-concentration $\left(S_{\mathrm{cc}}\right.$, squares) structure factors along the 111-direction for the occupation numbers $\left\langle n_{1}\right\rangle=10,\left\langle n_{2}\right\rangle=1$ are shown. The statistics for the simulation data is known to be poor in $k$-space.

figure 6 for the phase point $\left\langle n_{1}\right\rangle=10,\left\langle n_{2}\right\rangle=1$. The agreement is reasonable, although there are still fairly large statistical errors, a problem commonly encountered in direct calculations of $S(k)$ (as opposed to Fourier transforming $g(r)$ ). The error bars on the simulation results (not shown) are rather large.

In the calculations above we neglected all contributions from configurations in the partition sum (11) other than the (presumed) ground state, where all occupation numbers are the same. This is of course only justified when the other configurations have much higher energies. To check this hypothesis, we explore the energy landscape of small lattice systems with $\mu_{1}=\mu_{2}$. For a $2 \times 2 \times 2$ lattice systems, one can explicitly enumerate all local energy minima. In the case of no nearest-neighbour interactions the Boltzmann factor has $2^{8}$ degenerate local maxima. Slowly switching on nearest-neighbour interactions by setting $\boldsymbol{\eta}=\lambda \epsilon$ and varying $\lambda$, one can numerically follow the local maxima of the Boltzmann factor. Due to the high symmetry, many local minima stay degenerate even at finite nearest-neighbour interaction. When increasing $\boldsymbol{\eta}$, some local minima turn into saddle points and vanish, as illustrated in figure 7 . The remaining excited energy states depending on $\lambda$ are shown in the same figure. The increasing gap between the ground state and the excited states is clearly seen. For larger lattices the explicit enumeration of all local minima is no longer feasible because of their large number. We therefore resort to a simple Metropolis algorithm employed in numerical optimization problems [13]. Instead of systematically searching the $2 L$-dimensional occupation number state, we generate trial configurations randomly from the previously found local minimum, analogous to the random particle displacements used in Monte Carlo simulations. After each random step in the space of occupation numbers a local minimization of the energy is performed. The random step is then accepted or rejected based on the energy difference of the two local minima. Effectively the very rugged energy landscape is therefore replaced with a locally constant function, thereby eliminating the numerical problems one usually encounters when solving global optimization problems. In figure 8 the resulting local energy minima are shown together with the (presumed) homogeneous ground state. All the minima 


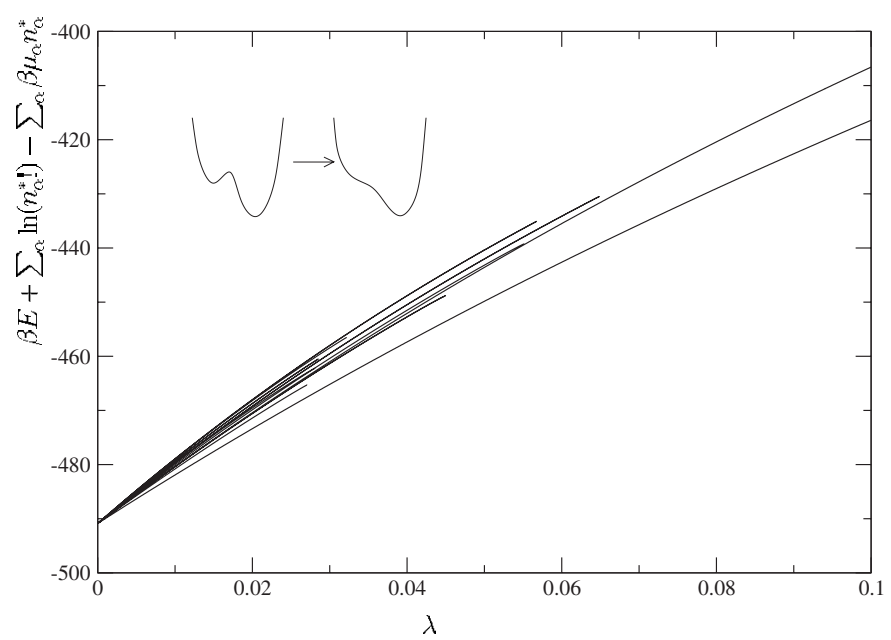

Figure 7. Local energy minima for a $2 \times 2 \times 2$ lattice system at $\mu_{1}=\mu_{2}=6.5 k_{B} T$. The energy minima are degenerate for vanishing nearest-neighbour interaction $(\lambda=0)$ and split for finite $\lambda$ into sets of local minima related by symmetry of the lattice. At certain critical $\lambda$, some minima turn into saddle points and vanish, as visualized in the inset.

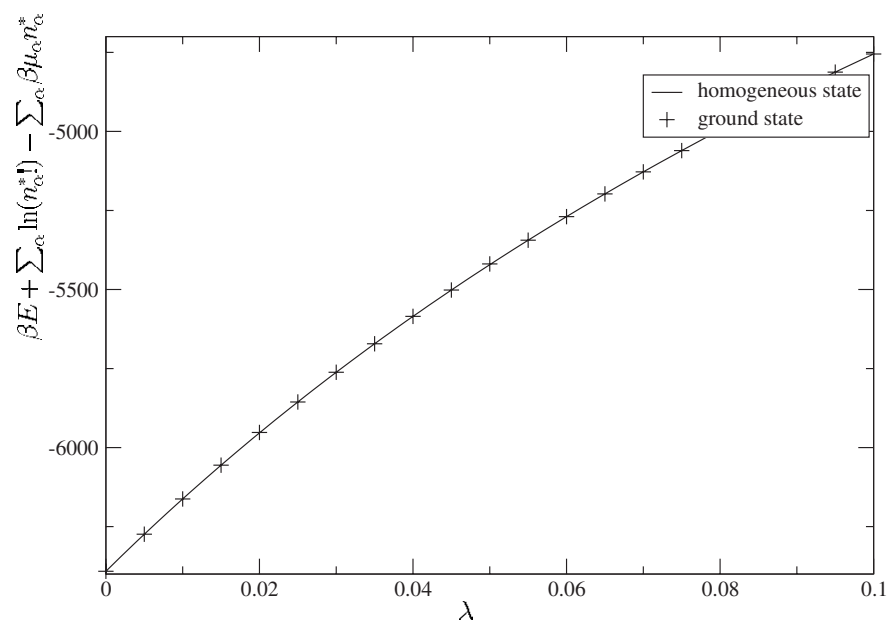

Figure 8. Absolute ground state energies in a $5 \times 5 \times 5$ lattice at $\beta \mu_{1}=\beta \mu_{2}=6.5$ as found by the optimization procedure described in the text (plusses) compared to the energy of the homogeneous state, as a function of the nearest-neighbour interaction $\boldsymbol{\eta}=\lambda \epsilon$. On site interactions are $\epsilon_{11}=\epsilon_{22}=0.1 k_{B} T, \epsilon_{12}=0.2 k_{B} T$.

lie on or slightly above the presumed ground state, thereby supporting our hypothesis that the ground state is the homogeneous state. In figure 9 the difference between the local minima and the ground state energy is plotted. Even though some local minima are not found during the search, one can clearly see a trend of an increasing gap between the ground state and the first excited state when the nearest-neighbour interaction increases. For a nearest neighbour interaction that equals $10 \%$ of the on-site interaction one finds a gap of approximately $1.5 k_{B} T$. 


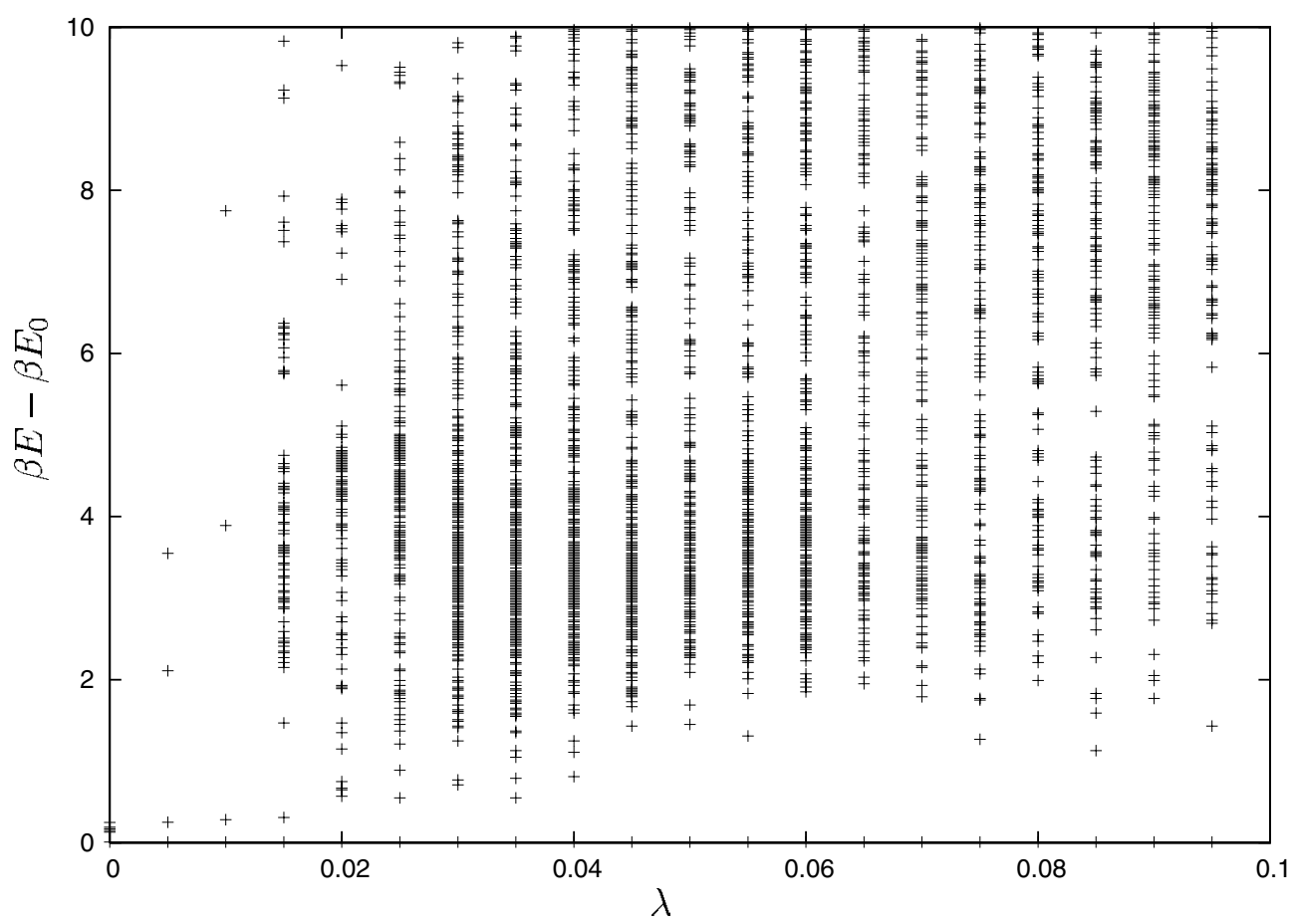

Figure 9. Local energy minima with respect to the ground state, as found by the optimization procedure described in the text. The on site interactions are $\epsilon_{11}=\epsilon_{22}=0.1 k_{B} T, \epsilon_{12}=0.2 k_{B} T$. The nearest-neighbour interactions are $\boldsymbol{\eta}=\lambda \epsilon$ with $\lambda=0,0.005,0.01, \ldots, 0.1$. It is clearly seen that the energy gap from the ground state to the first excited state increases with increasing nearest-neighbour interaction. The ground-state approximation should therefore become more accurate for stronger nearest-neighbour interaction.

Even stronger nearest-neighbour interaction should further widen the gap, leading to a higher accuracy of the ground-state approximation.

\section{Gibbs ensemble simulations}

To test the validity of the ground state approximation, Gibbs ensemble Monte Carlo simulations [15] of the model have been performed on a $10 \times 10 \times 10$ lattice. After an equilibration time of 1000000 steps two lattices were allowed to exchange particles and random moves within each lattice were attempted according to the standard metropolis algorithm. The simulations were restricted to symmetric interactions with $\epsilon_{11}=\epsilon_{22}$ and $\eta_{11}=\eta_{22}$. Since in these systems the equation of state must be invariant with respect to relabelling of the species (or equivalently mirroring the concentration $x \rightarrow 1-x$ ), volume changes of the lattice were not necessary, i.e. the two lattice systems were automatically under equal pressure. Two typical phase diagrams are shown in figures 10 and 11. It is seen that the ground-state approximation predicts the correct shape of the phase diagram, albeit underestimates the densities of the coexisting phases. The discrepancy becomes larger for larger interaction energies, when the contributions of the excited states are no longer negligible and the ground-state approximation becomes less accurate. 


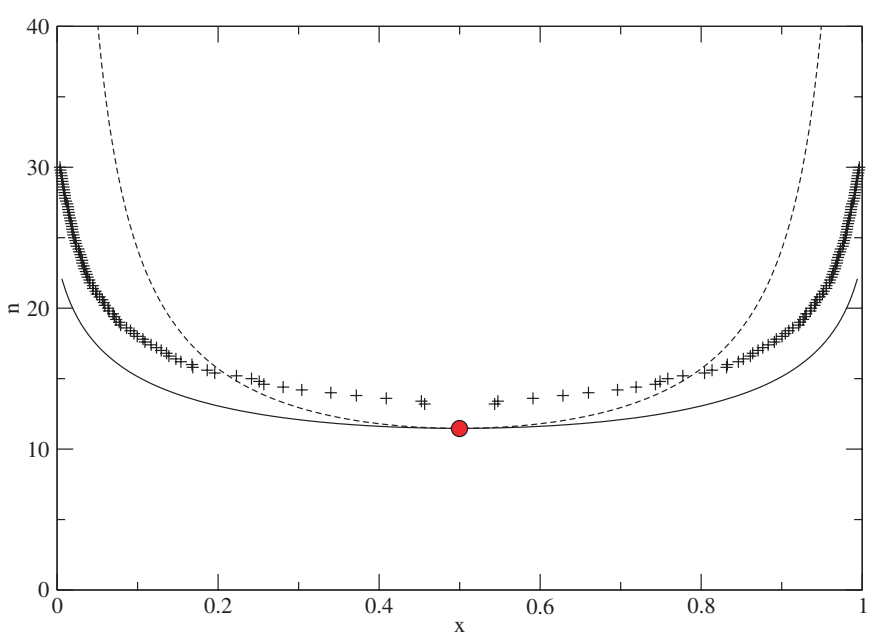

Figure 10. Phase diagram of a symmetric binary lattice system with $\epsilon_{11}=\epsilon_{22}=0.1 k_{B} T, \epsilon_{12}=$ $0.2 k_{B} T, \eta=0.1 \epsilon$. The phase coexistence points of the Gibbs ensemble simulation (crosses) and the predictions of the binodal (solid line) and spinodal (dashed line) based on the ground-state approximation are shown. No microphase separation is expected for such low nearest-neighbour interactions.

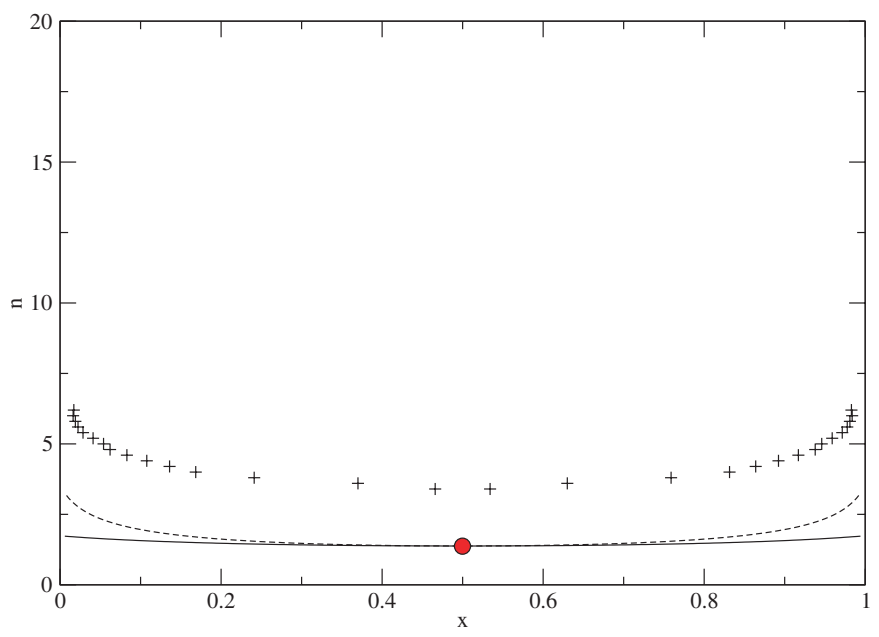

Figure 11. Phase diagram of a symmetric binary lattice system with $\epsilon_{11}=\epsilon_{22}=0.5 k_{B} T, \epsilon_{12}=$ $1 k_{B} T, \eta=0.1 \epsilon$ as in figure 10 .

\section{Conclusion}

The lattice model introduced in this paper, which allows us for multiple occupancy of lattice sites by one or two species, with positive energy penalties, and includes repulsive nearestneighbour interactions, leads to interesting phase behaviour. In the one-component version, a steepest descent analysis allowing for Gaussian fluctuations, predicts microphase separation onto two interpenetrating sub-lattices with different mean occupation numbers ('checkered' phase) when the ratio $\eta / \epsilon$ of nearest-neighbour to on-site couplings exceeds a threshold. This prediction is confirmed by Monte Carlo simulations. 
The two-component extension of the model leads to a macroscopic demixing transition into phases of different concentrations of the two species. The steepest descent analysis predicts such a phase separation, even in the absence of nearest-neighbour interactions, but this is shown to be due to the expected break-down of the approximation. In the presence of nearest-neighbour interactions, the steepest-descent analysis corresponds to a 'groundstate' approximation. A numerical search of local minima of the energy surface points to a significant gap $\left(>1 k_{B} T\right)$ between the homogeneous 'ground state' and the first 'excited states', thus providing support for the validity of the approximation. The predicted phase diagrams agree reasonably well with the results of Gibbs ensemble MC simulations. The present model is directly inspired by the continuous 'Gaussian core' model, first introduced by Stillinger [6], and which has recently been shown to provide an adequate coarse-grained description of interacting, interpenetrating polymer coils [3]. The corresponding lattice model, considered here, appears to have some unorthodox features. In contrast to more familiar lattice gas models, it is not isomorphous to an Ising spin model. Hence it is not yet clear whether its critical behaviour belongs to the Ising universality class. The binary version is expected to exhibit both the microphase separation found here in the one-component case, and the macroscopic phase separation analogous to that of the continuous binary Gaussian core model [8-10]. The interplay between these two phase transitions should lead to interesting and novel behaviour, which will be explored in future work.

\section{Acknowledgments}

RF would like to thank the Oppenheimer fund for financial support. AAL wishes to acknowledge the Royal Society for their funding. The authors are grateful to Jonathan Doye for valuable discussions and for pointing out [13] to us.

\section{References}

[1] Grosberg A Y, Khalatur P G and Khokhlov A R 1982 Makromol. Chem. Rapid Commun. 3709

[2] Dautenhahn J and Hall C K 1994 Macromolecules 275399

[3] Louis A A, Bolhuis P G, Hansen J P and Meijer E J 2000 Phys. Rev. Lett. 852522

Bolhuis P G, Louis A A, Hansen J.-P and Meijer E J 2001 J. Chem. Phys. 1144296

[4] Likos C N, Löwen H, Watzlawek M, Abbas B, Jucknischke O, Allgaier J and Richter D 1998 Phys. Rev. Lett. 804450

[5] Groot R D and Warren P B 1997 J. Chem. Phys. 1074423

[6] Stillinger F 1976 J. Chem. Phys. 653968

[7] Lang A, Likos C N, Watzlawek M and Löwen H 2000 J. Phys.: Condens. Matter 125087 Likos C N, Lang A, Watzlawek M and Löwen H 2001 Phys. Rev. E 631206

[8] Louis A A, Bolhuis P G and Hansen J-P 2000 Phys. Rev. E 627961

[9] Archer A J and Evans R 2001 Phys. Rev. E 64041501

[10] Finken R, Hansen J P and Louis A A 2003 J. Stat. Phys. 1101015

[11] Baker G A, Jr Bishop A R, Fesser K, Beale P D and Krumhansl J A 1982 Phys. Rev. B 262596

[12] Likos C N, Watzlawek M and Löwen H 1998 Phys. Rev. E 583135

[13] Wales D J and Doye J P K 1997 J. Phys. Chem. A 101511

[14] Bhatia A B and Thornton D E 1970 Phys. Rev. B 23004

[15] Frenkel D and Smit B 2002 Understanding Molecular Simulation 2nd edn (New York: Academic) 